OPEN ACCESS

Edited by:

Timothy Michael Ellmore,

The City College of New York, USA

Reviewed by:

Miriam Gade,

Catholic University of

Eichstatt-Ingolstadt, Germany

Nick Duran,

Arizona State University, USA

${ }^{*}$ Correspondence:

Stefan Scherbaum

Department of Psychology,

Technische Universität Dresden

Zellescher Weg 17, 01062 Dresden,

Germany

stefan.scherbaum@tu-dresden.de

Specialty section:

This article was submitted to

Cognition,

a section of the journal

Frontiers in Psychology

Received: 30 April 2015 Accepted: 22 June 2015 Published: 06 July 2015

Citation:

Scherbaum S, Gottschalk C, Dshemuchadse $M$ and Fischer $R$

(2015) Action dynamics in multitasking: the impact of additional task factors on the execution of the prioritized motor movement.

Front. Psychol. 6:934.

doi: 10.3389/fpsyg.2015.00934

\section{Action dynamics in multitasking: the impact of additional task factors on the execution of the prioritized motor movement}

\author{
Stefan Scherbaum *, Caroline Gottschalk, Maja Dshemuchadse and Rico Fischer \\ Department of Psychology, Technische Universität Dresden, Dresden, Germany
}

In multitasking, the execution of a prioritized task is in danger of crosstalk by the secondary task. Task shielding allows minimizing this crosstalk. However, the locus and temporal dynamics of crosstalk effects and further sources of influence on the execution of the prioritized task are to-date only vaguely understood. Here we combined a dual-task paradigm with an action dynamics approach and studied how and according to which temporal characteristics crosstalk, previously experienced interference and previously executed responses influenced participants' mouse movements in the prioritized task's execution. Investigating continuous mouse movements of the prioritized task, our results indicate a continuous crosstalk from secondary task processing until the endpoint of the movement was reached, although the secondary task could only be executed after finishing execution of the prioritized task. The motor movement in the prioritized task was further modulated by previously experienced interference between the prioritized and the secondary task. Furthermore, response biases from previous responses of the prioritized and the secondary task in movements indicate different sources of such biases. The bias by previous responses to the prioritized task follows a sustained temporal pattern typical for a contextual reactivation, while the bias by previous responses to the secondary task follows a decaying temporal pattern indicating residual activation of previously activated spatial codes.

Keywords: action dynamics, mouse movements, crosstalk, dual task, task shielding, cognitive control, conflict adaptation

\section{Introduction}

Multitasking seems to be ubiquitous in today's world. The execution of multiple tasks at the same time, however, runs the risk of the prioritized task's performance being affected by the additional tasks. For example, even highly practiced and prioritized driving performance might suffer from additional task execution (e.g., Levy et al., 2006; Strayer and Drews, 2007).

In the present study it was thus asked to which extent continuous motor movements of a prioritized task are affected by determinants of a multitasking context (e.g., programming of a subsequently executed motor task). To study how a prioritized task (e.g., Task 1: a number magnitude judgment) is influenced by simultaneous processing of additional task components (e.g., Task 2: a tone frequency judgment) most experiments use dual-task 
paradigms in which (1) the stimulus (S2) of Task 2 is presented in various intervals (stimulus onset asynchronies, SOA) after the stimulus (S1) of Task 1 (Pashler, 1994) and (2) both tasks share dimensional overlap (Navon and Miller, 2002). In these settings, many studies reported that programming of the response (R1) in Task 1 is affected by simultaneous programming of the response (R2) in Task 2, reflected in so-called crosstalk effects. For example, Hommel (1998a) demonstrated crosstalk effects on RT in Task 1 (RT1) when the response codes of Task 1 and 2 overlapped, i.e., responding to colors in Task 1 with a left or right key-press and to letters in Task 2 by saying "left" and "right." In this case, RT1 decreased when Task 2 indicated the same response category, while it increased when Task 2 indicated a different response category.

Although the amount of between-task interference, reflected in crosstalk effects, has often been taken to indicate the effectiveness of cognitive control in shielding the prioritized task processing i.e., small crosstalk effects reflecting strong task shielding (Logan and Gordon, 2001; Fischer and Hommel, 2012; Plessow et al., 2012; Fischer et al., 2014), the locus and temporal dynamics of crosstalk effects are to date only vaguely understood. In addition, while most studies investigated how response programming in one task affects response programming in another, only few studies targeted execution-related interference between tasks. For example, Bratzke et al. (2009) used continuous motor movements in Task 1 and found propagation effects of movement distance in Task 1 on choice RT in Task 2, indicating Task 1 motor execution-related interference in Task 2. In a similar setup, Ulrich et al. (2006) found that prolonging response-execution in Task 1 also increased choice RT on Task 2. While these studies investigated effects of continuous motor movements on additional task processing, we pursued the opposite approach by focusing on the quality of the prioritized continuous motor execution and crosstalk effects due to additional task processing on these movements. More specifically, we applied a crosstalk approach to test in which time windows and by which factors the continuous motor execution of a prioritized Task 1 is affected. For this we designed a dynamic dual-task paradigm, in which participants had to move a computer mouse to respond in both tasks. Importantly, a continuous analysis of mouse movements allows first, to track accuracy/quality of the movement parameter and second to determine the temporal characteristics of the influencing factors (Spivey et al., 2005; Freeman et al., 2008; Scherbaum et al., 2010).

We hypothesized at least three important factors to determine motor execution in Task 1. First, we predict that simultaneous programming of an additional motor response affects the execution of the prioritized motor movement: interference and hence deviations of the prioritized movement could be expected if the additional motor response points into a different direction as the prioritized movement. Participants responded to the magnitude of a presented number (S1) by moving the mouse to a pre-defined target region (see Figure 1). To ensure R2 programming while executing Task 1, S2 (high vs. low tone) was presented shortly with different SOAs following S1. Both tasks were performed sequentially using the same response device. R1 was given by moving the mouse toward target regions at the upper left and the upper right of the screen, while R2 was given by subsequently moving the mouse toward target regions at the lower left and the lower right of the screen. The brief presentation of S2 required encoding and possibly programming of R2. Yet, the execution of R2 was not to start until execution of R1 was completed. If R2 programming starts prior to completion of $\mathrm{R} 1$ execution, programming $\mathrm{R} 2$ that entails opposite directional movement parameters (e.g., a spatial code for the target region on the right side of the screen) should critically affect the quality of the continuous motor execution of R1 (e.g., movement in direction of the left target region). Importantly, and in contrast to previous crosstalk studies employing discrete button presses, the continuous performance measure allows determining whether and to which time point $\mathrm{R} 2$ programming affects the movement

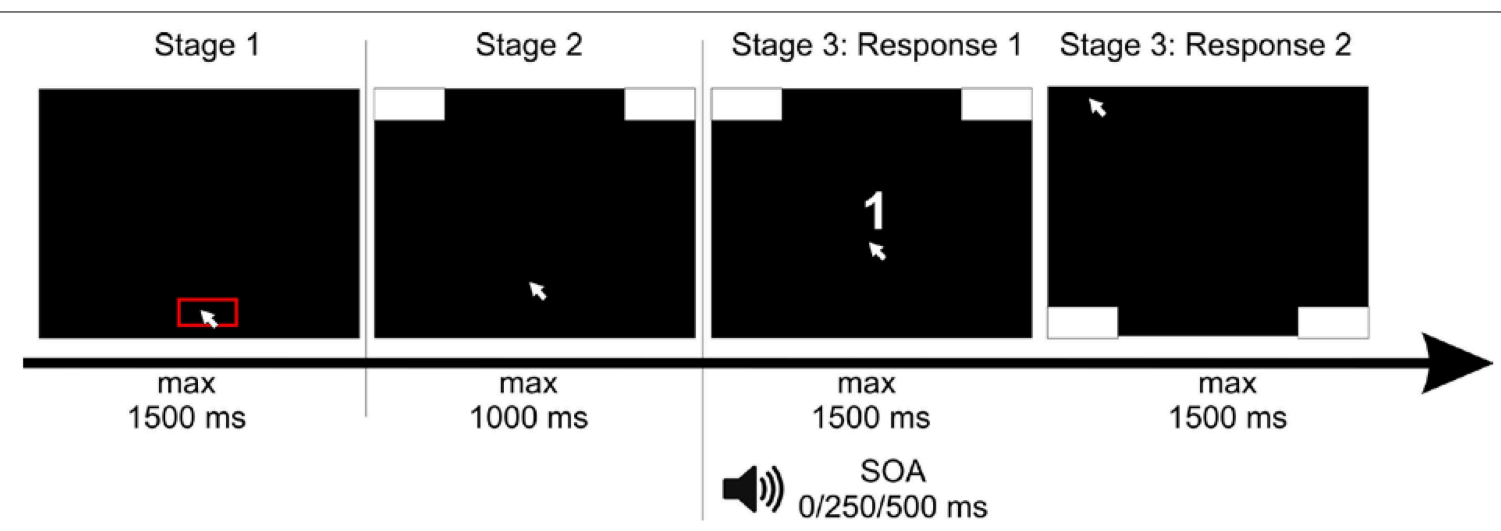

FIGURE 1 | Setup of the experiment: Participants had to click with the mouse cursor into a red box at the bottom of the screen. After clicking, response boxes appeared at the upper edge of the screen and participants had to move the cursor upwards, in order to start the trial. After reaching a movement threshold, the stimulus of the first task $-\mathrm{a}$ white number-appeared. The second stimulus, a tone, was presented with a stimulus onset asynchrony (SOA) of 0/250/500 ms. For the response to the first stimulus, participants had to move the mouse cursor to the left or the right upper response box as indicated by the number. Afterwards - for the response to the second stimulus-participants had to move the mouse cursor to the left or the right bottom response box as indicated by the tone. 
of R1. This is not trivial, as findings from single tasks indicate time-sensitive profiles of interference. Whereas, in the Simon task interference effects decrease over time due to either decay or suppression of conflicting information (Hommel, 1994; Stürmer et al., 2002; Band et al., 2003; Scherbaum et al., 2010), in the Stroop task and the Flanker task, the opposite temporal pattern was observed (Pratte et al., 2010; Ulrich et al., 2015). In addition, R1 movement execution might reach a ballistic dynamic in the direction of the aimed target region at which it might become immune to influences of additional Task 2 processing.

Second, in a recent study, we found that previously executed responses influenced motor movement parameter in the current trial (Scherbaum et al., 2010). At the start of a trial, participants showed a movement bias in the direction of the previously executed response. In the present dual-task situation, two responses from the previous trial could influence Task 1 execution: the previous response to Task 1 and the previous response to Task 2 , respectively. Because the movement pattern of R2 (downward movement) differs substantially to the movement pattern of R1 (upward movement), a bias on the current R1 by pervious R2 could be driven by residual activation of the spatial code (left vs. right target region) of the previous $\mathrm{R} 2$. This residual activation should drop off quickly after response execution and hence, one could expect this influence to decay quickly.

Given the similarity of previous and current R1 (both upward movements) we assume that a bias may consist of the reactivation of the entire motor program including the previously targeted spatial code. This reactivation of the previously applied motor program should be reflected in a more sustained influence on the current response to Task 1 . Both influences and the described temporal patterns can be detected by the analysis of mouse movements.

As a third influential parameter on current prioritized movement execution we hypothesize that the extent of betweentask interference in the previous trial (i.e., the level of crosstalk in trial $_{\mathrm{N}-1}$ ) will determine shielding of the currently executed motor response from crosstalk. This assumption is derived from the influential conflict monitoring theory (Botvinick et al., 2001) which proposes that an experienced response conflict triggers a recruitment of cognitive control to optimize subsequent performance. As a consequence, interference effects are usually reduced when following a conflict (Gratton et al., 1992; Stürmer et al., 2002; Egner and Hirsch, 2005; Ullsperger et al., 2005). In a dual-task context, crosstalk interference from Task 2 onto Task 1 processing can be interpreted as conflict that in turn shows sequential dependencies (e.g., Fischer et al., 2014). Hence, it is conceivable that the present form of crosstalk in a continuous motor execution task leads to similar sequential modulations of interference. Demonstrating trial-totrial modulations of dual-task specific crosstalk effects extends the idea of conflict adaptation to yet another form of conflict. This is not trivial, as conflicts in single tasks usually contain taskirrelevant features/stimuli that can be suppressed by mechanisms of selective attention. Dual-task processing, however, differs considerably as all features/stimuli are task-relevant and a simple selective attention mechanism might not be adaptive (see also Fischer et al., 2014).
Furthermore, by applying continuous motor movements to study these modulations in dual-task situations, our study extends previous studies investigating temporal dynamics of congruence sequence effects (e.g., Notebaert et al., 2006; Mayr and Awh, 2009; Egner et al., 2010)-however, the study of continuous movements enables a within-trial approach yielding precise temporal patterns by which previous interference affects current Task 1 execution.

\section{Methods}

\section{Participants}

Twenty students (17 female, mean age $=23.52$ years, $S D=$ 4.41) of the Technische Universität Dresden took part in the experiment ${ }^{1}$. All participants had normal or corrected to normal vision. They received class credit or $5 €$ payment.

\section{Ethics Statement}

The study was approved by the institutional review board of the Technische Universität Dresden and conducted in accordance to ethical standards of the 1964 Declaration of Helsinki and of the German Psychological Society. All participants were informed about the purpose and the procedure of the study and gave written informed consent prior to the experiment. All data were analyzed anonymously.

\section{Apparatus and Stimuli}

Target stimuli in Task 1 (numbers 1-4 and 6-9) were presented in white on a black background in the center of on a 17 inch screen running at a resolution of $1280 \times 1024$ pixels $(75 \mathrm{~Hz}$ refresh frequency). S1 had a width of $6.44^{\circ}$. Response boxes $\left(11.55^{\circ}\right.$ in width) in Task 1 were presented at the top left and top right of the screen. S2 were sine tones (low: $440 \mathrm{~Hz}$, high: $880 \mathrm{~Hz}$, sampled at $44,100 \mathrm{~Hz}$ ), presented for $200 \mathrm{~ms}$ binaurally via headphones. Response boxes ( $11.55^{\circ}$ in width) in Task 2 were presented at the bottom left and bottom right of the screen.

For presentation, we used Psychophysics Toolbox 3 (Brainard, 1997; Pelli, 1997), Matlab 2006b (the Mathworks Inc.), and Windows XP. Tones were presented via the Portaudio driver on high precision ASIO enabled soundcards. Responses were carried out by moving a computer mouse (Logitech Wheel Mouse USB), sampled with a frequency of $92 \mathrm{~Hz}$.

\section{Procedure}

After onscreen instructions and demonstration by the experimenter, participants practiced 20 trials, followed by the main experiment. The experiment consisted of four blocks and 1028 trials overall (see Design).

Each trial consisted of three stages (see Figure 1). In the first stage, participants had to click at a red box $\left(11.55^{\circ}\right.$ in width $)$ at the bottom of the screen within a deadline of $1500 \mathrm{~ms}$. This served to produce a comparable starting area for each trial. After clicking within this box, the second stage started and two response boxes at the right and left upper corner of the screen were presented.

\footnotetext{
${ }^{1}$ Descriptive data of one student participant was lost after data collection-hence, gender and age describe data of 19 participants. The experimental data are reported completely for all 20 participants.
} 
Participants were required to start the mouse movement upwards within a deadline of $1500 \mathrm{~ms}$. We chose this procedure forcing participants to be already moving when entering the decision process to assure that they did not decided first and then only executed the final movement. Hence, only after moving at least 4 pixels in each of two consecutive time steps, the third stage started containing the actual tasks one and two. The target stimulus of Task 1-the number-was presented. The stimulus of Task 2the tone-was presented with a stimulus onset asynchrony (SOA) of 0,250 , or $500 \mathrm{~ms}$ relative to $\mathrm{S} 1$.

To minimize "noise" in the data (due to incompatible stimulus-space representations (e.g., Dehaene et al., 1993), we used a constant spatial-compatible mapping between stimuli and responses for all participants. More precisely, for the first task, participants were instructed to respond to the number by moving the cursor into the upper left response box for digits smaller than five and to the upper right response box for digits larger than five. After giving this response to Task 1, participants executed their response to Task 2 by moving the cursor into the bottom left response box for a low tone and to the bottom right response box for the high tone. The number and the tone either indicated the same side of response (congruent condition) or opposite sides (incongruent condition).

The trial ended after moving the cursor into the respective response boxes or within a response deadline of $1500 \mathrm{~ms}$ in each task (see Figure 1). If participants missed deadlines of one of the three stages, the next trial started with the presentation of the red start box. Response times (RT) were measured as the time to reach the respective response box, reflecting the interval between the onset of the target stimulus (number in the first task, tone in the second task) and reaching the response box (top ones for the first task, bottom ones for the second task) with the mouse cursor.

\section{Design}

Across trials, we varied the following independent variables: for the current trial, number $_{N}(1,2,3,4,6,7,8$, and 9) and tone $N$ (low/high), and for the previous trial, number $_{N-1}$ and tone $e_{N-1}$. The sequence of trials was balanced within each block by pseudo randomization resulting in a balanced $\operatorname{trial}_{N}(16) \times$ trial $_{N-1}(16)=256$ trials transition matrix $(+1$ trial to conclude the sequence of balanced transitions) for each of four blocks of trials, resulting in 1028 trials overall. On this balanced sequence of trials, the SOA between number and tone was distributed balanced across congruency ${ }_{N}$ and congruency $y_{N-1}$ by pseudo randomization. Overall, this leads to a $2\left(\right.$ congruency $\left.y_{N}\right) \times$ $2\left(\right.$ congruenc $\left._{N-1}\right) \times 3(S O A)$ design with 85-86 trials per condition.

\section{Data Preprocessing}

We excluded trials missing deadlines or containing erroneous responses and the trial following erroneous responses in one or both of the two tasks (15.47\%). For all analyses of discrete measures, Greenhouse-Geisser adjustments were applied when appropriate.

For the analysis of mouse movements, we aligned all movements for a common starting position and normalized each movement to 100 equal time slices ${ }^{2}$ (Spivey et al., 2005; Scherbaum et al., 2010). To quantify the deviation of mouse movements from the shortest path to the target box, we subtracted $\mathrm{X}$-coordinates of each movement from an ideal Xcoordinate line.

\section{Results}

\section{Response Times in Task 1 (RT1) ${ }^{3}$}

We first analyzed the impact of between-task crosstalk in the current trial (congruency ) $_{\text {) }}$ and the between-task crosstalk of

\footnotetext{
${ }^{2}$ At $92 \mathrm{~Hz}$ sampling frequency, 100 samples correspond to an RT of $1087 \mathrm{~ms}$. This means that all trials below this RT, including trials of average RT ( $M=682 \mathrm{~ms}$, $S E=19 \mathrm{~ms}, 62$ samples at $92 \mathrm{~Hz}$ ), are stretched to 100 samples. Only trials longer than $1087 \mathrm{~ms}$ ( $5.7 \%$ of all trials) are compressed.

${ }^{3}$ For RT2, please see the Supplementary Material and the Supplementary Figure 1.
}

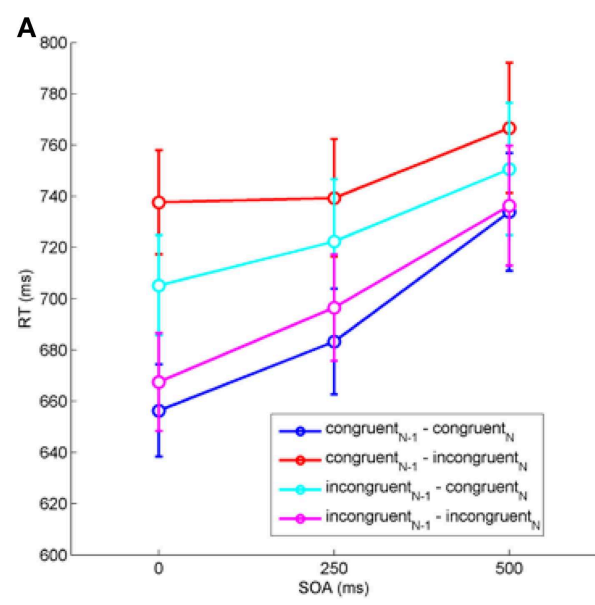

FIGURE 2 | (A): RT1 as a function of stimulus onset asynchrony (SOA in ms) between the stimulus of Task 1 and Task 2. (B): Results of multiple regression analysis on RT1 (in $\mathrm{ms}$ ) with all hypothesized factors. Negative

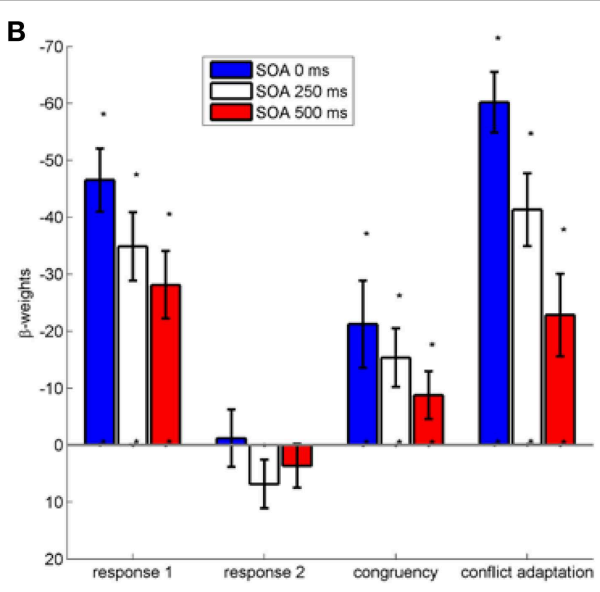

weights indicate a decrease in RT. Error bars represent standard errors of the mean. Asterisks indicate significant differences from zero at the 0.05 level (see main text). 
the previous trial (congruency ${ }_{N-1}$ ) on RT1 (see Figure 2A). A repeated measures analysis of variance (ANOVA) on RT1 with the factors congruency $y_{N}$, congruency $y_{N-1}$, and SOA revealed significant crosstalk from Task 2 to Task 1 as expressed in the main effect of congruency $y_{N}$ on RT1, $F_{(1,19)}=11.61, p<$ $0.01, \eta_{p}^{2}=0.38$. RT1 were shorter when the present trial was congruent (709 ms) than incongruent (724 ms). Post-conflict trials were slightly faster $(713 \mathrm{~ms})$ compared to trials following congruent trials $(720 \mathrm{~ms})$ as indicated by the significant factor congruency $_{N-1}, F_{(1,19)}=6.32, p<0.05, \eta_{p}^{2}=0.25$. A main effect of $S O A$ indicated larger RT1 $(692,710,747 \mathrm{~ms})$ with increasing SOA, $F_{(2,38)}=33.52, p<0.001, \eta_{p}^{2}=0.64$.

Furthermore, there was a significant two-way interaction congruency $_{N} \mathrm{x}$ congruency $y_{N-1}, F_{(1,19)}=77.53, p<0.001, \eta_{p}^{2}=$ 0.8 , reflecting a conflict adaptation effect that was also present for all individual SOA levels (individual ANOVAs for each $\mathrm{SOA}$, all $p s<0.01)$. Yet, the significant three-way interaction between congruency $y_{N} \times$ congruency $_{N-1} \times S O A$ on RT1 shows that the expression of conflict adaptation varied across SOA levels, $F_{(2,38)}=11.49, p<0.001, \eta_{p}^{2}=0.38$ (see Figure 2A). No other interactions reached statistical significance (all $p>0.3$ ).

To establish a comparison for the continuous regression analysis as performed on mouse movements (see next section), we studied the full pattern of hypothesized effects with respect to SOA by performing regression analysis on RT1. We performed regression separately for each SOA with regressors for all four hypothesized, namely congruency $y_{N}$, conflict adaptation (congruency $_{N} \times$ congruency $_{N-1)}$, first response in previous trial (current R1 as repetition or switch of previous R1), and second response in previous trial (current $\mathrm{R} 1$ as repetition or switch of previous R2). All regressors were normalized to a range of $[0,1]$. To exclude multicolinearity, we checked variance inflation factors to stay below 1 . We tested the resulting 12 beta-weights (3 SOA $\times$ 4 regressors) for statistical significant influence by $t$-tests against zero.

Results (see Figure 2B) show significant beta-weights across all SOA for congruency $y_{N}$ (all $\beta<-9$, all $t<-2.10$, all $p<0.05$ ), conflict adaptation (all $\beta<-23$, all $t<-3.154$, all $p<0.05$ ), and first response in previous trial (all $\beta<-28$, all $t<-4.760$, $p<0.05$ ), but not for second response in previous trial (all $p>0.12$ ). Hence, congruency within a trial, conflict adaptation, and a repetition of the previous response in Task 1 significantly influenced the response in the current trial across all SOA.

\section{Mouse Movements in Task 1}

In the next step we analyzed mouse movements to investigate the temporal patterns of the different influences in dependence of the SOA. To this end, we performed time continuous multiple regression (Notebaert and Verguts, 2007; Scherbaum et al., 2010; but see Mirman et al., 2008 for a multilevel approach) on the deflection of mouse movements on the (horizontal) X-axis (see Supplement Figure 2): For each trial, we calculated deflection as the difference of the real movement and a straight line from the start-point to the end-point of the real movement (a hypothetical direct movement). Compared to movements on the $\mathrm{X}$-axis, this measure removes random variance resulting from different start- and end-points of movements and instead focusses on the deviation of the movement away from an ideal movement due to influences during movement execution. Compared to movement-angles (Scherbaum et al., 2010), deviation is more robust to noise, as it integrates influences across time, though at the cost of temporal resolution.

For regression on this continuous measure of deflection, we used the same regressors as for RT1, namely congruency, conflict adaptation (congruency ${ }_{N} \times$ congruency $_{N-1}$ ), first response in previous trial (current $\mathrm{R} 1$ as repetition or switch of previous $\mathrm{R} 1$ ), and second response in previous trial (current $\mathrm{R} 1$ as repetition or switch of previous R2). For each time slice, we calculated a multiple regression analysis (100 time slices $\rightarrow 100$ multiple regressions analyses) with the four defined regressors, yielding four time-varying beta weights ( 4 weights across 100 time slices) for each participant. For each of these four beta-weights, we computed grand averages representing the time-varying strength of influence curve for each predictor. To detect significant temporal segments of influence, we calculated $t$-tests against zero for each time step of these beta-weights (Scherbaum et al., 2010; Dshemuchadse et al., 2012), compensating for multiple comparisons of temporally dependent data by only accepting segments of more than 10 consecutive significant $t$-tests (see Appendix for a Monte Carlo analysis on this issue, based on Dale et al., 2007).

As can be seen in Figure 3 and Table 1, congruency $y_{N}$ showed a significant influence across all SOAs and the temporal onset of significant influence by congruency ${ }_{N}$ strictly followed the SOA. Furthermore, conflict adaptation was only present for the first two SOAs, with a slight time-lag to the onset of congruency $_{N}$. A long lasting influence of first response in previous trial was present across all SOA, while second response in previous trial influenced mouse movements, in contrast to RT1, but only at the start of the trial, decaying quickly in the course of the trial ${ }^{4}$.

These results confirm our expectation that information from the previous tasks and from the current Task 2 influenced the execution of Task 1. Furthermore, crosstalk from Task 2 on Task 1 was not limited to a specific stage of Task 1 execution, but was present for all SOA and followed the timing of the arrival of information from Task 2 as reflected in the SOA.

\section{Discussion}

In the present study we investigated to which extent continuous motor movements of a prioritized task (Task 1) are affected by determinants of a multitasking context, namely crosstalk from a secondary task (Task 2), previously executed responses of Task 1 and Task 2, and the extent of previously experienced interference between Task 1 and Task 2 (crosstalk in trial N-1). We found evidence for an influence of all four factors, following specific temporal patterns.

First, the results from RT and mouse movements indicate that Task 1 execution is influenced by crosstalk through the information necessary to program the response of Task 2 (Hommel, 1998a; Logan and Schulkind, 2000; Fischer et al., ${ }^{4}$ These analyses did not change qualitatively when removing all trials with RT
lower than $500 \mathrm{~ms}$. 

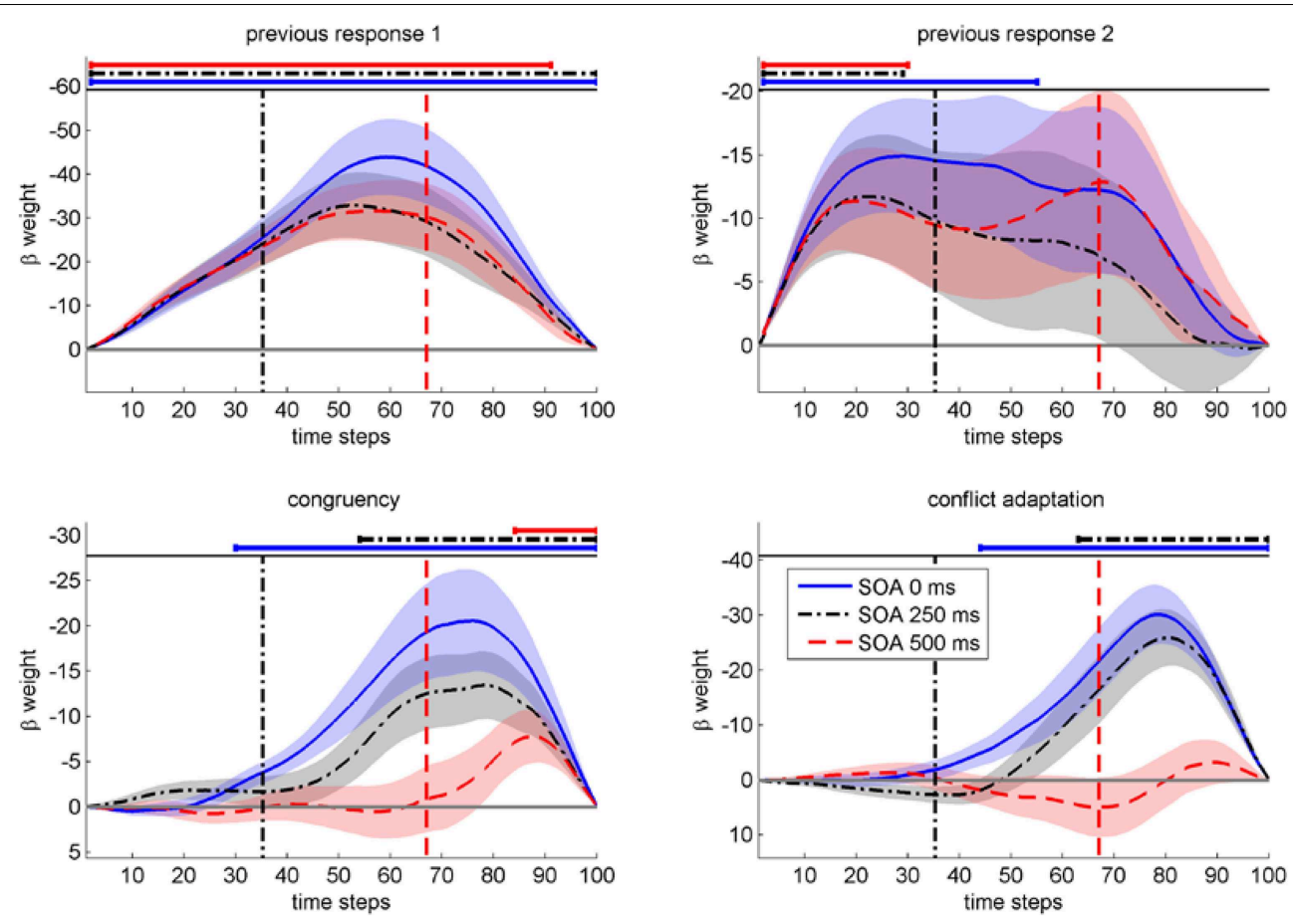

FIGURE 3 | Mean beta weights from continuous regression analysis on the deflection of mouse movements (in pixels) as a function of time steps (normalized time). Each regressor for each of three SOAs (in ms) is plotted in separate panels. The SOAs' time segment relative to

average RT1 is indicated by the respective vertical lines. Negative beta-weights indicate a support of movement into the correct direction (smaller deflection to the incorrect target box). Shaded areas indicate standard errors.

TABLE 1 | Significant temporal segments (normalized time) from continuous regression analysis and respective mean RT1.

\begin{tabular}{|c|c|c|c|c|}
\hline Regressor & & SOA $0 \mathrm{~ms}$ & SOA $250 \mathrm{~ms}$ & SOA $500 \mathrm{~ms}$ \\
\hline \multirow[t]{2}{*}{ Congruencyn } & Time steps & $30-100$ & $54-100$ & $84-100$ \\
\hline & $M(R T)$ & $207-691 \mathrm{~ms}$ & $383-710 \mathrm{~ms}$ & $627-746 \mathrm{~ms}$ \\
\hline \multirow[t]{2}{*}{ Conflict adapation } & Time steps & $44-100$ & $63-100$ & - \\
\hline & $M(R T)$ & $304-691 \mathrm{~ms}$ & $447-710 \mathrm{~ms}$ & - \\
\hline \multirow[t]{2}{*}{ First response $_{\mathrm{N}-1}$} & Time steps & $2-100$ & $2-100$ & $2-91$ \\
\hline & $\mathrm{M}(\mathrm{RT})$ & $14-691 \mathrm{~ms}$ & $14-710 \mathrm{~ms}$ & $15-679 \mathrm{~ms}$ \\
\hline \multirow[t]{2}{*}{ Second response $_{\mathrm{N}-1}$} & Time steps & $2-55$ & $2-29$ & $2-30$ \\
\hline & $\mathrm{M}(\mathrm{RT})$ & $14-380 \mathrm{~ms}$ & $14-206 \mathrm{~ms}$ & $15-224 \mathrm{~ms}$ \\
\hline
\end{tabular}

2007). While the analysis of RT1 indicated crosstalk to weaken with increasing SOA, the analysis of mouse movements revealed that crosstalk strictly followed the timing of the onset of the information for Task 2 as determined by the SOA. For the shortest SOA, the influence of crosstalk started the earliest and accumulated most in the deflection of mouse movements. For the longest SOA, the influence of crosstalk was limited to the final part of the movement and could accumulate only shortly. Thus, while varying in degree, crosstalk was not limited to specific critical time-windows that might be related to certain processing stages, i.e., response selection and/or movement execution of Task 1 . The finding of crosstalk on Task 1 movement execution is

not trivial. First of all, although the movement of the mouse itself started the trial (with $\mathrm{S} 1$ presentation) so that $\mathrm{R} 1$ programming was forced to occur online during movement, it could have been conceivable that the movement becomes a ballistic process at some point which renders it insensitive to influences of additional stimulus encoding and classification. In contrast, however, we could show that throughout the entire movement period crosstalk from additional Task 2 processing affected the movement quality in the prioritized task-even for the longest SOA, we found crosstalk, as indicated by regression analysis of RT1 and mouse movements. Furthermore, even though the execution of both tasks was temporally segregated (due to using the same response device for Task 1 and Task 2) crosstalk did occur whenever S2 was presented. This shows that the brief presentation of S2 resulted in an immediate stimulus feature encoding and response selection process that interfered with motor execution in Task 1. Put differently, despite the mouseparadigm-inherent sequential motor execution, crosstalk from Task 2 onto Task 1 could not be prevented. Since R1 and R2 movements were executed in different vertical directions (upwards for R1, downwards for R2), one could assume that the found crosstalk does not stem directly from the programming of movements, but from the spatial (horizontal) codes for the target areas (left/right), that overlapped between Task 1 and Task 2 .

Second, the analysis of RT1 and mouse movements yielded different results about the influence on R1 by previously executed responses in Task 1 and Task 2 . The analysis of RT1 only revealed an influence of the previous response in Task 1, while mouse 
movements revealed an influence of the previous response in both Task 1 and Task 2. This indicates that RT as a discrete measure was not as sensitive as mouse movements and missed the smaller effects of the previous R2. Mouse movements further revealed distinct temporal patterns for both influences. That is, the previous response to Task 1 led to a strong and sustained influence which can be interpreted as a retrieval of the previously activated response by the context of Task 1 (cf. Hommel, 1998b; Hommel et al., 2002). Since this effect was present across the whole trial, it was also reflected in $\mathrm{RT} 1$. The previous response to Task 2 led to a weaker and quickly decaying influence. This could be interpreted as a passively decaying residual activation of the response executed directly before the current R1. Notably, the differences in the exact movements of R1 (upwards) and R2 (downwards) suggests that this residual activation stems from spatial codes used for programming R2, but not from the completely programmed movement of the previous R2 itself. Since the effect decayed quickly, it was not reflected in RT1, indicating the advantage of analyzing the continuous data. The finding of these two effects from previously executed responses also sheds light on a similar effect found in single task situations (Scherbaum et al., 2010, 2013): here, the strength of the found response repetition bias might result from an inseparable mixture of the retrieval and residual activation of the same response in the previous trial.

Third, congruence relations in the previous trial affected crosstalk in the current trial. More specifically, previous conflict reduced the current impact of Task 2 processing on Task 1 . Therefore, experiencing crosstalk resulted in increased levels of prioritized task shielding to protect Task 1 processing from Task 2 interference that could be compared to conflict adaptation in single task situations (Botvinick et al., 2001).

Our findings support the view of a continuous process of scheduling and capacity sharing (Tombu and Jolicour, 2003). The crosstalk between Task 1 and 2 and the influence of previous interference indicate flexible task shielding of Task 1 from Task 2 following a pattern similar to conflict adaptation in single task situation (Botvinick et al., 2001). The effects of conflict adaptation indicate that task shielding can be parameterized by previous experience of crosstalk interference (Fischer et al., 2014; compare e.g., Logan and Gordon, 2001). However, adjustments to task shielding showed a time-lag leading to conflict adaptation in mouse movements being only present for the first two SOAs. For the longest SOA, the temporal pattern shows an onset of conflict adaptation that fails to reach significance before the end of R1.

The continuous nature of our task might have supported the flexible time sharing compared to the usual key-press-based setups. We forced participants to start the movement of R1 before S1 was presented and this could have forced participants to choose a continuous processing mode that might not be chosen in a key-press based paradigm. While this procedure was necessary to ensure that response selection is reflected in the movement of $\mathrm{R} 1$, one could also argue that most actions in the real world demand the continuous adaptation of response movements to occurring stimuli and hence, our results imply a higher ecological validity compared to the strongly constrained key-press setups found in other studies.
Notably, our study is not the first one to apply continuous movements to respond to Task 1 . However, previous studies used the continuous movements in Task 1 mainly to influence responding in Task 2 (e.g., Ulrich et al., 2006; Bratzke et al., 2008, 2009). An important variable in these studies was movement distance in Task 1, leading to higher movement times of R1. Especially for long movement distances, these studies found increasing Task 1 RT in dependence of the SOA, comparable to the results of the current study (although focusing on the consequences on Task 2; e.g., Bratzke et al., 2009).

A negative side-effect of our focus on Task 1 and the chosen response setup of our paradigm is that responses in Task 2 are hard to interpret. In the case of incongruent Task 1 and Task 2 responses, participants had a longer way to reach the opposite response box in Task 2 and hence longer RT per se. However, what could be taken from the pattern of RT in Task 1 and Task 2 is that our manipulation of SOA was effective. If higher SOA had shown smaller slopes in RT of Task 2 (see Supplement), it would have been possible that Task 2 information was too late to influence Task 1 (compare e.g., Ulrich et al., 2006).

Concluding, the dynamic investigation of Task 1 execution in a dual-task setting yielded three findings: First, the crosstalk from Task 2 interfered with Task 1 execution. Although this influence was clearly dependent on the temporal proximity between S1 and S2 presentation, the impact of the influence onto Task 1 processing was continuous, i.e., irrespective of any critical windows of influence. This indicates a continuous process of task execution that does not end in a ballistic automatic movement, but is prone to interference until reaching its final destination; second, the modulation of crosstalk by previous interference indicates a flexible adaptation of task-shielding; and third, the execution of Task 1 was also influenced by previously executed responses of both, Task 1 and Task 2however, these influences showed different temporal patterns indicating a sustained reactivation of the previous response of Task 1 and a decaying residual activation that is most likely related to the spatial codes of the previous execution of Task 2.

\section{Author Contributions}

This research was supported by the German Research Foundation (DFG grant SCH1827/11 to SS and DFG grant SFB 940/1 Project A3 to RF). The funders had no role in study design, data collection and analysis, decision to publish, or preparation of the manuscript. No additional external funding was received for this study.

\section{Funding}

We acknowledge support by the German Research Foundation and the Open Access Publication Funds of the TU Dresden.

\section{Supplementary Material}

The Supplementary Material for this article can be found online at: http://journal.frontiersin.org/article/10.3389/fpsyg. 2015.00934 


\section{References}

Band, G. P. H., Ridderinkhof, K. R., and Van Der Molen, M. W. (2003). Speedaccuracy modulation in case of conflict: the roles of activation and inhibition. Psychol. Res. 67, 266-279. doi: 10.1007/s00426-002-0127-0

Botvinick, M. M., Braver, T. S., Barch, D. M., Carter, C. S., and Cohen, J. D. (2001). Conflict monitoring and cognitive control. Psychol. Rev. 108, 624-652. doi: 10.1037/0033-295X.108.3.624

Brainard, D. H. (1997). The psychophysics toolbox. Spat. Vis. 10, 433-436. doi: $10.1163 / 156856897$ X00357

Bratzke, D., Rolke, B., and Ulrich, R. (2009). The source of execution-related dualtask interference: motor bottleneck or response monitoring? J. Exp. Psychol. Hum. Percept. Perform. 35, 1413-1426. doi: 10.1037/a0015874

Bratzke, D., Ulrich, R., Rolke, B., Schröter, H., Jentzsch, I., and Leuthold, H. (2008). Motor limitation in dual-task processing with different effectors. Q. J. Exp. Psychol. 61, 1385-1399. doi: 10.1080/17470210701536856

Dale, R., Kehoe, C., and Spivey, M. J. (2007). Graded motor responses in the time course of categorizing atypical exemplars. Mem. Cognit. 35, 15-28. doi: 10.3758/BF03195938

Dehaene, S., Bossini, S., and Giraux, P. (1993). The mental representation of parity and number magnitude. J. Exp. Psychol. Gen. 122, 371-396. doi: 10.1037/00963445.122.3.371

Dshemuchadse, M., Scherbaum, S., and Goschke, T. (2012). How decisions emerge: action dynamics in intertemporal decision making. J. Exp. Psychol. Gen. 142, 151-185. doi: 10.1037/a0028499

Egner, T., Ely, S., and Grinband, J. (2010). Going, going, gone: characterizing the time-course of congruency sequence effects. Front. Cogn. 1:154. doi: $10.3389 /$ fpsyg. 2010.00154

Egner, T., and Hirsch, J. (2005). The neural correlates and functional integration of cognitive control in a Stroop task. Neuroimage 24, 539-547. doi: 10.1016/j.neuroimage.2004.09.007

Fischer, R., Gottschalk, C., and Dreisbach, G. (2014). Context-sensitive adjustment of cognitive control in dual-task performance. J. Exp. Psychol. Learn. Mem. Cogn. 40, 399-416. doi: 10.1037/a0034310

Fischer, R., and Hommel, B. (2012). Deep thinking increases task-set shielding and reduces shifting flexibility in dual-task performance. Cognition 123, 303-307. doi: 10.1016/j.cognition.2011.11.015

Fischer, R., Miller, J., and Schubert, T. (2007). Evidence for parallel semantic memory retrieval in dual tasks. Mem. Cognit. 35, 1685-1699. doi: 10.3758/BF03193502

Freeman, J. B., Ambady, N., Rule, N. O., and Johnson, K. L. (2008). Will a category cue attract you? Motor output reveals dynamic competition across person construal. J. Exp. Psychol. Gen. 137, 673-690. doi: 10.1037/a0013875

Gratton, G., Coles, M. G., and Donchin, E. (1992). Optimizing the use of information: strategic control of activation of responses. J. Exp. Psychol. Gen. 121, 480-506. doi: 10.1037/0096-3445.121.4.480

Hommel, B. (1994). Spontaneous decay of response-code activation. Psychol. Res. 56, 261-268. doi: 10.1007/BF00419656

Hommel, B. (1998a). Automatic stimulus-response translation in dual-task performance. J. Exp. Psychol. Hum. Percept. Perform. 24, 1368-1384. doi: 10.1037/0096-1523.24.5.1368

Hommel, B. (1998b). Event files: evidence for automatic integration of stimulusresponse episodes. Vis. cogn. 5, 183-216. doi: 10.1080/713756773

Hommel, B., Müsseler, J., Aschersleben, G., and Prinz, W. (2002). The theory of event coding (TEC): a framework for perception and action planning. Behav. Brain Sci. 24, 849-878. doi: 10.1017/S0140525X01000103

Levy, J., Pashler, H., and Boer, E. (2006). Central interference in driving is there any stopping the psychological refractory period? Psychol. Sci. 17, 228-235. doi: 10.1111/j.1467-9280.2006.01690.x

Logan, G. D., and Gordon, R. D. (2001). Executive control of visual attention in dual-task situations. Psychol. Rev. 108, 393-434. doi: 10.1037/0033295X.108.2.393

Logan, G. D., and Schulkind, M. D. (2000). Parallel memory retrieval in dualtask situations: I. Semantic memory. J. Exp. Psychol. Hum. Percept. Perform. 26, 1072-1090. doi: 10.1037/0096-1523.26.3.1072

Mayr, U., and Awh, E. (2009). The elusive link between conflict and conflict adaptation. Psychol. Res. 73, 794-802. doi: 10.1007/s00426-008-0191-1
Mirman, D., Dixon, J. A., and Magnuson, J. S. (2008). Statistical and computational models of the visual world paradigm: growth curves and individual differences. J. Mem. Lang. 59, 475-494. doi: 10.1016/j.jml.2007.11.006

Navon, D., and Miller, J. (2002). Queuing or sharing? A critical evaluation of the single-bottleneck notion. Cogn. Psychol. 44, 193-251. doi: 10.1006/cogp.2001.0767

Notebaert, W., Gevers, W., Verbruggen, F., and Liefooghe, B. (2006). Top-down and bottom-up sequential modulations of congruency effects. Psychon. Bull. Rev. 13, 112-117. doi: 10.3758/BF03193821

Notebaert, W., and Verguts, T. (2007). Dissociating conflict adaptation from feature integration: a multiple regression approach. J. Exp. Psychol. 33, 1256-1260. doi: 10.1037/0096-1523.33.5.1256

Pashler, H. (1994). Dual-task interference in simple tasks: data and theory. Psychol. Bull. 116, 220-244. doi: 10.1037/0033-2909.116.2.220

Pelli, D. G. (1997). The VideoToolbox software for visual psychophysics: transforming numbers into movies. Spat. Vis. 10, 437-442. doi: $10.1163 / 156856897$ X00366

Plessow, F., Schade, S., Kirschbaum, C., and Fischer, R. (2012). Better not to deal with two tasks at the same time when stressed? Acute psychosocial stress reduces task shielding in dual-task performance. Cogn. Affect. Behav. Neurosci. 12, 557-570. doi: 10.3758/s13415-012-0098-6

Pratte, M. S., Rouder, J. N., Morey, R. D., and Feng, C. (2010). Exploring the differences in distributional properties between Stroop and Simon effects using delta plots. Atten. Percept. Psychophys. 72, 2013-2025. doi: 10.3758/APP.72.7.2013

Scherbaum, S., Dshemuchadse, M., Fischer, R., and Goschke, T. (2010). How decisions evolve: the temporal dynamics of action selection. Cognition 115, 407-416. doi: 10.1016/j.cognition.2010.02.004

Scherbaum, S., Dshemuchadse, M., Leiberg, S., and Goschke, T. (2013). Harder than expected: increased conflict in clearly disadvantageous intertemporal choices in a computer game. PLOS ONE 8:e79310. doi: 10.1371/journal.pone.0079310

Spivey, M. J., Grosjean, M., and Knoblich, G. (2005). Continuous attraction toward phonological competitors. Proc. Natl. Acad. Sci. U.S.A. 102, 10393-10398. doi: 10.1073/pnas.0503903102

Strayer, D. L., and Drews, F. A. (2007). Cell-phone-induced driver distraction. Curr. Dir. Psychol. Sci. 16, 128-131. doi: 10.1111/j.1467-8721.2007. 00489.x

Stürmer, B., Leuthold, H., Soetens, E., Schroter, H., and Sommer, W. (2002). Control over location-based response activation in the Simon task: behavioral and electrophysiological evidence. J. Exp. Psychol. Hum. Percept. Perform. 28, 1345-1363. doi: 10.1037/0096-1523.28.6.1345

Tombu, M., and Jolicœur, P. (2003). A central capacity sharing model of dualtask performance. J. Exp. Psychol. Hum. Percept. Perform. 29, 3-18. doi: 10.1037/0096-1523.29.1.3

Ullsperger, M., Bylsma, L. M., and Botvinick, M. M. (2005). The conflict-adaptation effect: it's not just priming. Cogn. Affect. Behav. Neurosci. 5, 467-472. doi: 10.3758/CABN.5.4.467

Ulrich, R., Fernández, S. R., Jentzsch, I., Rolke, B., Schröter, H., and Leuthold, H. (2006). Motor limitation in dual-task processing under ballistic movement conditions. Psychol. Sci. 17, 788-793. doi: 10.1111/j.1467-9280.2006.01783.x

Ulrich, R., Schröter, H., Leuthold, H., and Birngruber, T. (2015). Automatic and controlled stimulus processing in conflict tasks: superimposed diffusion processes and delta functions. Cogn. Psychol. 78, 148-174. doi: 10.1016/j.cogpsych.2015.02.005

Conflict of Interest Statement: The authors declare that the research was conducted in the absence of any commercial or financial relationships that could be construed as a potential conflict of interest.

Copyright ( 2015 Scherbaum, Gottschalk, Dshemuchadse and Fischer. This is an open-access article distributed under the terms of the Creative Commons Attribution License (CC BY). The use, distribution or reproduction in other forums is permitted, provided the original author(s) or licensor are credited and that the original publication in this journal is cited, in accordance with accepted academic practice. No use, distribution or reproduction is permitted which does not comply with these terms. 